\title{
Long-term changes in keratometry and refraction after small aperture corneal inlay implantation
}

This article was published in the following Dove Press journal:

Clinical Ophthalmology

\author{
Majid Moshirfar ${ }^{1,2}$ \\ Jordan D Desautels ${ }^{1,3}$ \\ Brian D Walker ${ }^{4}$ \\ Orry C Birdsong' \\ David F Skanchy ${ }^{4}$ \\ Tyler S Quist ${ }^{5}$ \\ Michael S Murri ${ }^{6}$ \\ Steve H Linn' \\ Phillip C Hoopes Jr ${ }^{1,2}$ \\ Phillip C Hoopes ${ }^{1,2}$
}

'Hoopes, Durrie, Rivera Research Center, Hoopes Vision, Draper, UT, USA; ${ }^{2}$ John A. Moran Eye Center, Department of Ophthalmology and Visual Sciences, School of Medicine, University of Utah, Salt Lake City, UT, USA; ${ }^{3}$ The Warren Alpert Medical School, Brown University, Providence, RI, USA; ${ }^{4}$ McGovern Medical School, The University of Texas Health Science Center at Houston, Houston, TX, USA; ${ }^{5}$ Department of Ophthalmology and Visual Sciences, Carver College of Medicine, University of lowa, lowa City, IA, USA; ${ }^{6}$ Baylor College of Medicine, Houston, TX, USA

Correspondence: Majid Moshirfar Hoopes, Durrie, Rivera Research Center, Hoopes Vision, I I820S State Street, Suite \#200, Draper, UT 84020, USA

$\mathrm{Tel}+\mathrm{I} 8015680200$

Fax + I 80 I5630200

Email cornea2020@me.com
Purpose: To assess longitudinal refractive, keratometric, and topographic changes following KAMRA small-aperture inlay implantation.

Design and setting: Prospective study at a single site refractive surgery center.

Methods: Fifty patients underwent KAMRA small-aperture corneal inlay implantation for the correction of presbyopia. Uncorrected near visual acuity (UNVA), uncorrected distance visual acuity, manifest refractive spherical equivalent (MRSE), mean keratometry $(\mathrm{Km})$, corneal topography, and surgically induced astigmatism vector analysis assessments were performed preoperatively and at $1,3,6,12,24$, and 36 months postoperatively.

Results: The study comprises 50 eyes. An average shift of $0.15 \pm 0.63 \mathrm{D}$ (range -1.63 to $2.00 \mathrm{D}$ ) occurred between preoperative baseline and 36 months. At 36 months, $54 \%$ of patients had hyperopic MRSE and 40\% had myopic MRSE compared with baseline. Km was significantly elevated at all postoperative measurements compared with baseline, with the largest $\mathrm{Km}$ measured at 12 months. Eighty-six percent of patients had UNVA of 20/32 or better and $88 \%$ uncorrected distance visual acuity of $20 / 25$ or better at 36 months. Longitudinal corneal topography revealed a pattern of corneal steepening over the body of the inlay and flattening over the aperture, correlating with a hyperopic shift. There was no significant surgically induced astigmatism.

Conclusion: KAMRA inlay may cause an increase in Km compared with baseline. Corneal steepening may occur in a specific pattern with steepening over the inlay and flattening over the aperture. This topographic pattern causes a hyperopic shift, which may be relevant for subsequent procedures, such as cataract extraction.

Keywords: presbyopia, KAMRA, wound healing, IOL calculation

\section{Introduction}

Presbyopia affects approximately 1 billion individuals worldwide, with projections of increasing prevalence alongside an aging population. ${ }^{1,2}$ In recent years, corneal inlays, such as the KAMRA small-aperture inlay (AcuFocus, Irvine, CA, USA), have become alternatives to monovision and spectacle correction for the treatment of presbyopia.

Food and Drug Administration (FDA) clinical studies and independent literature have shown KAMRA to have a good safety, efficacy, and stability profile in a majority of patients. ${ }^{3-7}$ However, $73 \%$ of patients experienced blurred and fluctuating vision beyond 6 months postoperatively in the FDA trials. ${ }^{7}$ Moreover, a total of $8.5 \%$ of inlays were explanted [79.1\% of the explants were due to visual complaints: hyperopic shift $(55.8 \%)$, inadequate visual benefit $(16.3 \%)$, myopic shift $(4.7 \%)$, induced cylinder $(2.3 \%)]$. Given that the KAMRA inlay itself does not possess any refractive power, it is important to determine a mechanism of these refractive changes, beyond expected transient visual fluctuations related to surgery and postoperative drugs. 
Previous reports have suggested that poor visual results following KAMRA implantation may correlate with a localized region of corneal steepening directly over the inlay body, as well as corneal flattening over the aperture, along with corneal haze. ${ }^{8,9}$ These previous studies do not support a causal link between the observed pattern of steepening and the visual changes. This is the first study to our knowledge that simultaneously describes longitudinal visual, keratometric, and topographic changes following KAMRA inlay implantation in a large patient cohort.

\section{Methods}

A total of 65 emmetropic patients aged 45-60 years underwent KAMRA corneal inlay implantation for the correction of presbyopia between November and December 2009. The study was approved by the Hoopes, Durrie, Rivera (HDR) Research Review Board (Draper, Utah), and all data were collected in accordance with the tenets of the Declaration of Helsinki from patients who provided written informed consent to have their data used for research purposes. Inclusion in this study was contingent upon a diagnosis of presbyopia, preoperative spherical equivalents between $-0.75 \mathrm{D}$ and $0.50 \mathrm{D}$ with $<0.75 \mathrm{D}$ of refractive cylinder, uncorrected near visual acuity (UNVA) between 20/40 and 20/100 in the treatment eye, and a required reading addition of $+1.00 \mathrm{D}$ to $+2.50 \mathrm{D}$. Patients with abnormal topography, severe dry eye syndrome, active infection, herpetic eye complications, keratoconus, uncontrolled glaucoma, uncontrolled diabetes, active autoimmune disease, or connective tissue disease were excluded from this study.

Assessments were performed preoperatively and at 1 , $3,6,12,24$, and 36 months postoperatively for all 50 eyes. UNVA and uncorrected distance visual acuity (UDVA) were obtained with the Optec 6500 vision tester (Stereo Optical Inc., Chicago, IL, USA). Subjective refractions were obtained by the same optometrist (SHL). Steep and flat keratometry magnitudes and axes were established using the Pentacam HR Tomography (Oculus, Wetzler, Germany). Mean keratometry $(\mathrm{Km})$ denotes the average of the steep and flat corneal powers. Instantaneous (tangential) steepness maps were acquired with the RT-7000 Auto Ref-Topographer (Tomey Corporation, Nagoya, Japan).

\section{Surgical technique}

All surgeries were performed at a single site. The KAMRA inlay device used in this study has been previously described in detail. ${ }^{10}$ Inlays were implanted in an intrastromal pocket that was created with a $150 \mathrm{kHz}$ IntraLase femtosecond laser with a spot energy of $0.55-0.75 \mu \mathrm{J} /$ pulse and a spot/ line separation of $4 / 4 \mu \mathrm{m}$ (iFS; Abbott Medical Optics Inc., Santa Ana, CA, USA). Pocket parameters were as follows: corneal depth of $210 \mu \mathrm{m}$, channel width of $4.7 \mathrm{~mm}$, and a side-cut radius of $5.66 \mathrm{~mm}$. All side cuts were placed temporally. Inlays were centered on the Purkinje reflex when the reflex was $\leq 300 \mu \mathrm{m}$ from the pupil center and halfway between the pupil center and Purkinje reflex if the separation was $>300 \mu \mathrm{m}$. Postoperative care included a topical fluoroquinolone and Pred Forte (prednisolone acetate 1.0\%) four times daily for the first week followed by a 3-week taper. Preservative-free artificial tears were prescribed hourly for the first month and bottled tears every 2 to 3 hours for the following 2 months.

\section{Vector analysis}

Changes in corneal (keratometric) and refractive (manifest) astigmatism between successive postoperative time points and between the beginning and end of the follow-up period were computed using Alpins vector analysis software written in MATLAB (MathWorks Inc., Natick, MA, USA). ${ }^{11}$ Prior to Alpins analysis, all astigmatism measurements were transformed into positive cylinder notation. The Alpins method has been previously well described. In brief, changes in astigmatism can be represented as a vector that describes the magnitude and direction of astigmatic change between any two time points. The vector change before and after a surgical event is specifically referred to as the surgically induced astigmatism (SIA). The magnitude of the corneal astigmatism vector is described by the difference between the steep and flat keratometry readings (K2-K1), and the axis corresponds to the steep keratometric axis. Corneal astigmatism refers solely to the anterior corneal surface, whereas refractive astigmatism includes the contributions of the posterior cornea and crystalline lens to overall astigmatism.

Two definitions of the mean SIA vector are used in this article - the arithmetic vector mean and the summated vector mean. The arithmetic vector mean is the average of all SIA vector magnitudes without respect to direction, whereas the summated mean is computed as the head-to-tail sum of SIA vectors. Astigmatism and SIA vectors were plotted in polar coordinates for left and right eyes separately to account for temporal incision placement at $0^{\circ}$ and $180^{\circ}$, respectively. The arithmetic mean SIA is described for the entire patient population without stratification into left and right eye groups due to the fact that it is not dependent on vector direction. 


\section{Statistical analysis}

Statistical analyses were performed using SPSS software (version 24.0; SPSS Inc., Chicago, IL, USA). Km and manifest refractive spherical equivalent (MRSE) data were tested for normality using a Kolmogorov-Smirnov test to justify the use of paired Student's $t$-tests with Bonferroni correction for multiple tests. Statistical significance was determined by a $P$-value $<0.008$. The Pearson product-moment test was used for correlation analysis.

\section{Results}

Twelve patients were lost to follow-up and three patients were excluded due to lack of postoperative data. Of the 50 eyes included in this prospective study, 37 (74\%) were those of female and 13 (26\%) male. Preoperative patient demographics are summarized in Table 1. This study comprises 50 mean MRSE and Km values for the 50 study eyes that are summarized in Table 2. MRSE trended significantly toward myopia ( $t=4.138, P<0.0001)$ in the first postoperative month before undergoing a significant hyperopic shift between 1 and 3 months ( $t=-3.197, P=0.002$ ) (Figure 1). Consistent, but not statistically significant hyperopic shifts occurred in each postoperative interval prior to a minor myopic shift between 24 and 36 months. Overall, study eyes were significantly hyperopic with respect to baseline by 24 months ( $t=-2.895, P=0.006)$. Although statistically insignificant when measured in the group as a whole, by 36 months, $27 / 50(54 \%)$ of eyes exhibited some degree of hyperopic shift from baseline, with 13/27 (48\%) of these changes being clinically significant ( $\geq 0.5$ D). By contrast, 20/50 (40\%) of eyes had some degree of myopic shift from baseline, with $7 / 20(35 \%)$ being clinically significant. Additionally, 10/20 $(50 \%)$ of the eyes with an overall myopic shift experienced a clinically significant hyperopic shift at some point during the follow-up period; $3 / 50(0.6 \%)$ of eyes experienced no net change in MRSE by 36 months (Figure 2). Of note, $86 \%$ of

Table I Demographics of the study population

\begin{tabular}{ll}
\hline Characteristics & Mean \pm SD, number, range \\
\hline Age (years) & $51.6 \pm 3.7$ (range 46 to 59$)$ \\
Gender (female:male) & $37: 13$ \\
MRSE (D) & $-0.018 \pm 0.229$ (range -0.375 to 0.375 ) \\
Manifest cylinder (D) & $0.285 \pm 0.223$ (range 0 to 0.75 ) \\
LogMAR UDVA & $0.05 \pm 0.09$ (range 0.00 to 0.40$)$ \\
LogMAR UNVA & $0.56 \pm 0.07$ (range 0.2 to 0.6 ) \\
\hline
\end{tabular}

Abbreviations: MRSE, manifest refractive spherical equivalent; UDVA, uncorrected distance visual acuity; UNVA, uncorrected near visual acuity.
Table 2 Mean MRSE and Km values for postoperative time points

\begin{tabular}{lllll}
\hline Time point & Mean \pm SD MRSE & Mean \pm SD Km & Kmin & Kmax \\
\hline Preoperative & $-0.018 \pm 0.229$ & $43.84 \pm 1.32$ & 41.20 & 46.10 \\
I month & $-0.197 \pm 0.363$ & $44.08 \pm 1.29$ & 41.60 & 46.40 \\
3 months & $0.038 \pm 0.582$ & $44.14 \pm 1.43$ & 41.50 & 48.20 \\
6 months & $0.090 \pm 0.501$ & $44.16 \pm 1.33$ & 41.60 & 46.50 \\
I 2 months & $0.150 \pm 0.444$ & $44.21 \pm 1.36$ & 41.50 & 46.70 \\
24 months & $0.204 \pm 0.566$ & $44.12 \pm 1.42$ & 41.10 & 46.90 \\
36 months & $0.130 \pm 0.623$ & $44.07 \pm 1.35$ & 42.40 & 46.70 \\
\hline
\end{tabular}

Abbreviations: $\mathrm{Km}$, mean keratometry; $\mathrm{Kmax}$, maximum keratometry; Kmin, minimum keratometry; MRSE, manifest refractive spherical equivalent.

patients had UNVA of 20/32 or better and 88\% UDVA of $20 / 25$ or better at 36 months.

$\mathrm{Km}$ significantly steepened in the first postoperative month $(t=6.544, P<0.0001)$, continued to steepen at all postoperative intervals prior to 12 months, and then flattened, albeit without statistical significance between 12 and 24 months ( $t=2.008, P=0.05$ ) (Figure 3 ). Overall, Km was significantly elevated at all time points with respect to baseline. The average change in $\mathrm{Km}$ with respect to baseline was $0.23 \pm 0.332 \mathrm{D}$ (range $-0.3 \mathrm{D}$ to $1.4 \mathrm{D}$ ) at 36 months (Figure 4).

Longitudinal corneal topography showed a recurrent pattern of steepening over the annulus of the inlay with simultaneous flattening over the aperture in many study subjects (representative sample shown in Figure 5). In general, myopic regression following a period of hyperopic shift was accompanied by disappearance of this ring-like topographical pattern, as is shown for a single patient in Figure 6. Two patients with pronounced myopic shift showed an inverse pattern on corneal topography wherein corneal steepening occurred centrally and corneal flattening occurred peripherally as exemplified in Figure 7A. Central steepening was associated with myopic shift, whereas annular steepening was associated with hyperopic shift (Figure 7B).

\section{Vector analysis}

The mean keratometric and manifest SIA vector magnitudes between the preoperative and 36-month time points for the entire surgical cohort were $0.52 \mathrm{D} \pm 0.31$ (range 0.10 to 1.70 ) and $0.66 \mathrm{D} \pm 0.44$ (range 0.11 to 1.96 ), respectively. Table 3 shows the summated mean SIA vectors for all consecutive postoperative intervals as determined by vector analysis. Overall, keratometric SIA was largest in the first postoperative month and became relatively stable thereafter. There was an induction of $0.35 \mathrm{D}$ of keratometric astigmatism directed at $70^{\circ}$ for left eye implants and $0.28 \mathrm{D}$ of astigmatism directed 


\section{Change in mean MRSE between postoperative time points}

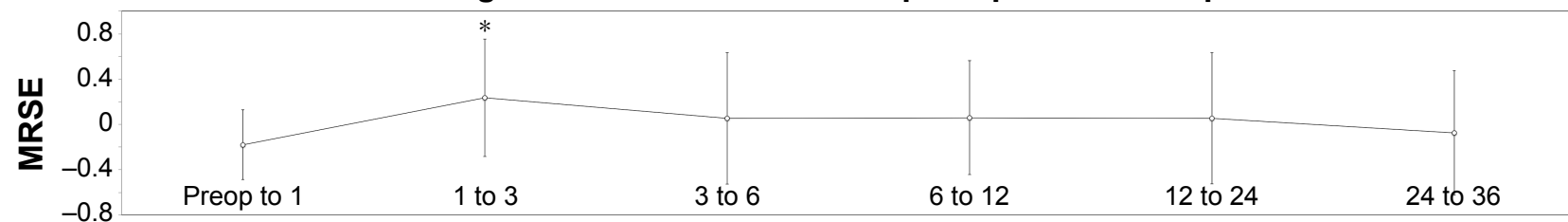

\section{Postoperative interval (months)}

Figure I Change in MRSE between postoperative time points for the entire patient cohort.

Note: *Statistically significant change.

Abbreviations: MRSE, manifest refractive spherical equivalent; preop, preoperative.

\section{Change in mean MRSE from baseline MRSE}

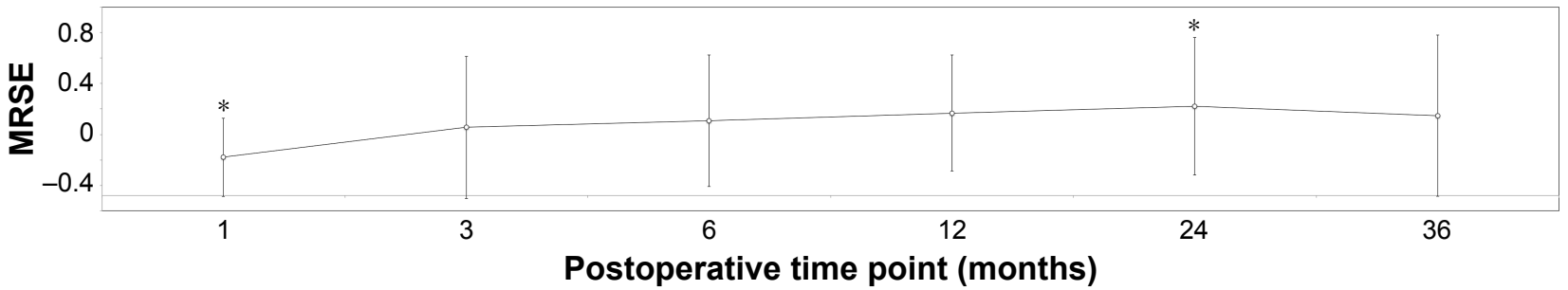

Figure 2 Change in MRSE with respect to baseline MRSE for all postoperative follow-up time points.

Note: *Statistically significant change.

Abbreviation: MRSE, manifest refractive spherical equivalent.

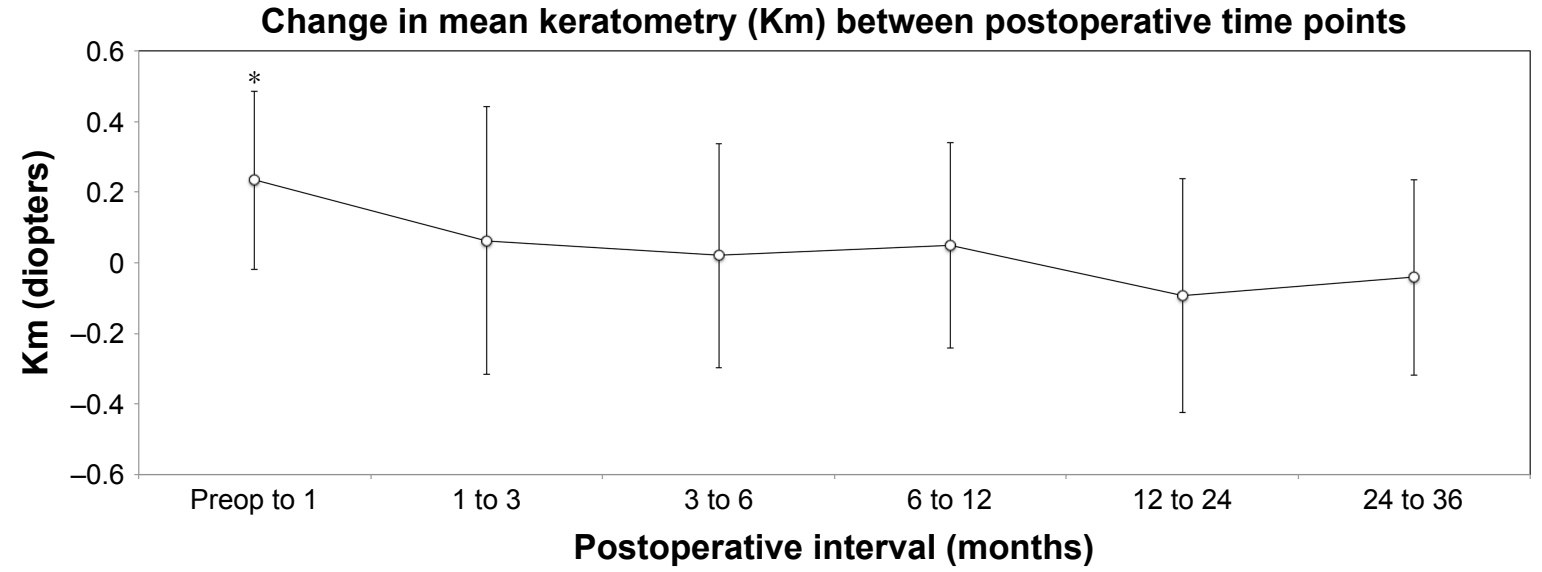

Figure 3 Change in $\mathrm{Km}$ between postoperative time points for the entire patient cohort.

Note: *Statistically significant change.

Abbreviations: $\mathrm{Km}$, mean keratometry; preop, preoperative.

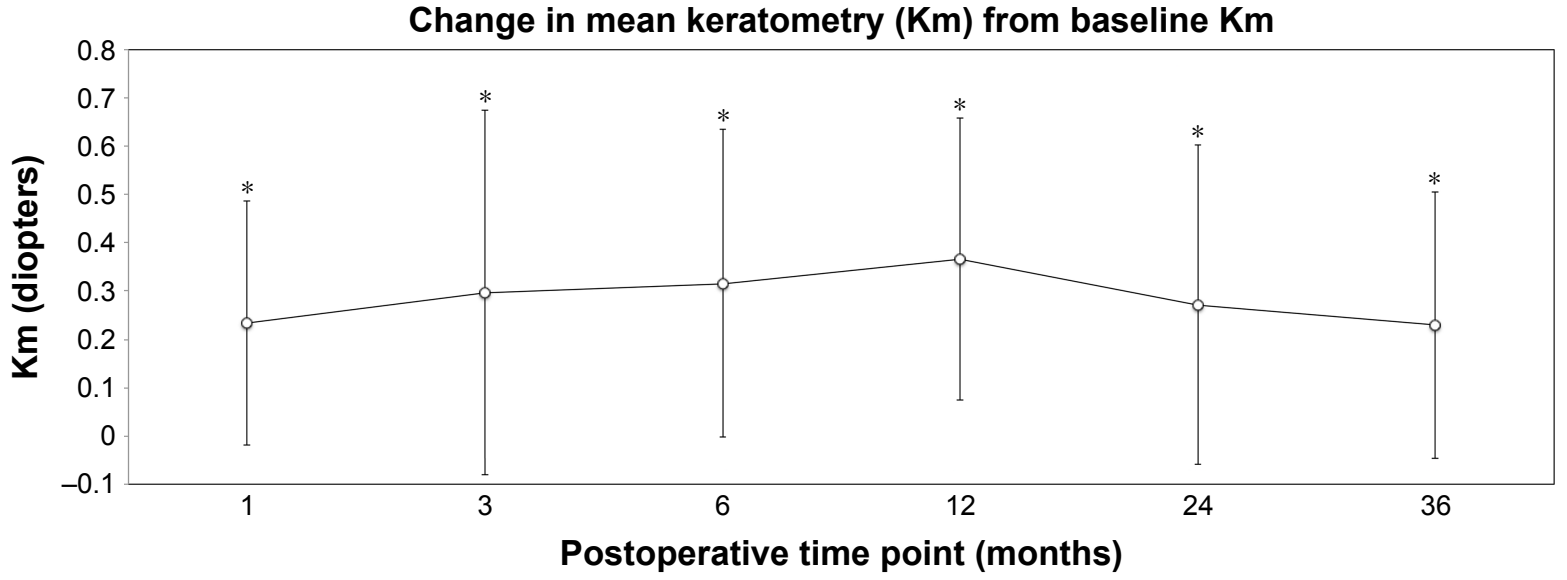

Figure 4 Change in $\mathrm{Km}$ with respect to baseline $\mathrm{Km}$ for all postoperative follow-up time points.

Note: *Statistically significant change.

Abbreviation: $\mathrm{Km}$, mean keratometry. 


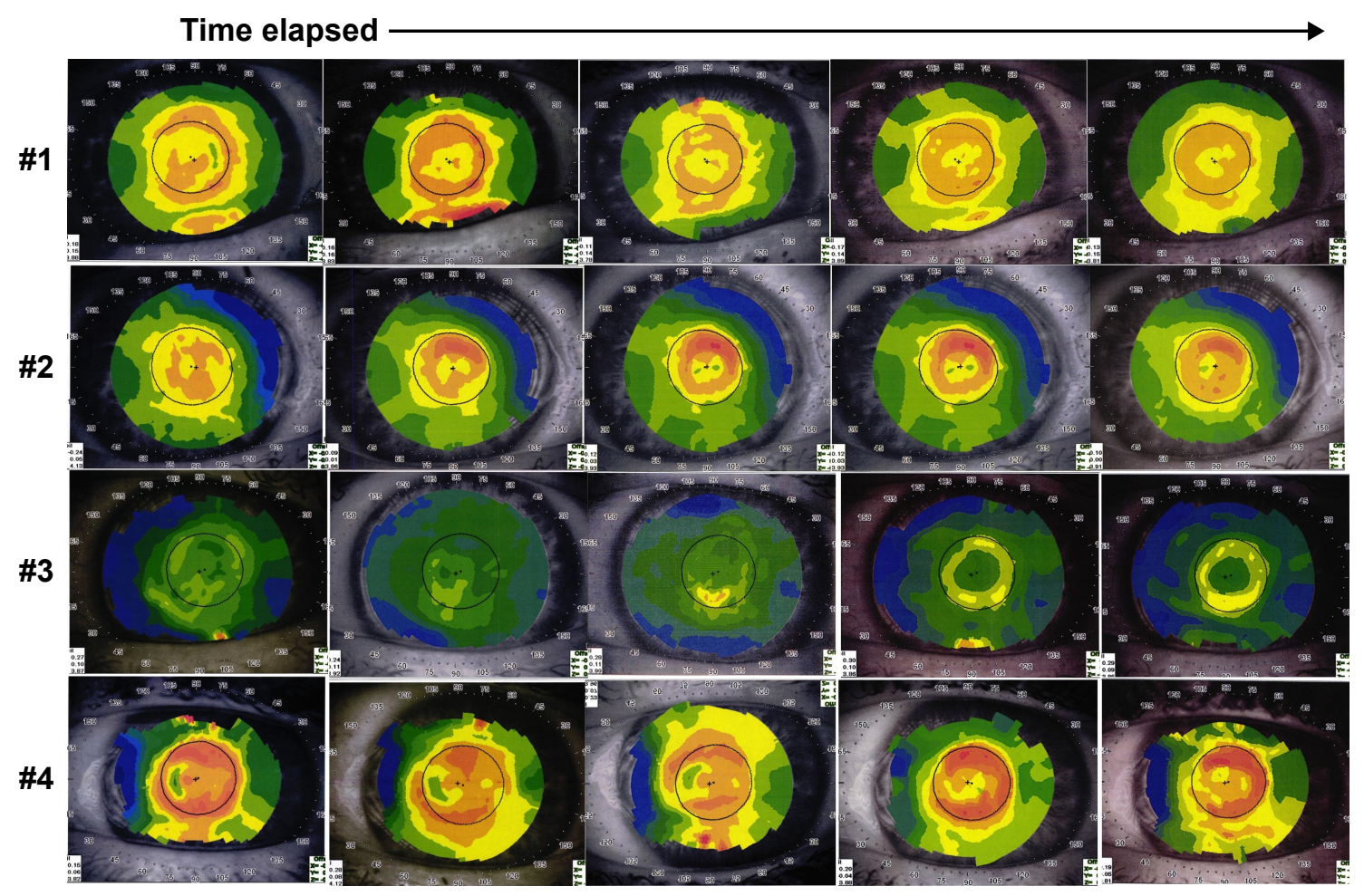

Figure 5 Demonstration of topographic pattern of annular steepening with central flattening occurring over the 36-month follow-up period with a corresponding hyperopic shift in four patients (\#I-4).

at $100^{\circ}$ for right eye implants by 36 months. Similarly, surgically induced manifest cylinder was $0.42 \mathrm{D}$ at $89^{\circ}$ among the left eye cohort and $0.35 \mathrm{D}$ at $106^{\circ}$ in the right eye cohort by 36 months (Table 3). Qualitatively, this represents an increase in corneal steepness at an axis similar to the mean preoperative astigmatism, as is visually represented in Figure 8. Keratometric and manifest SIA were not significantly different $(P=0.078)$ or correlated during this period $\left(R^{2}=0.001\right)$.

\section{Discussion}

As expected, our results demonstrated an inverse relation between $\mathrm{Km}$ and MRSE during the first postoperative month. Early myopic shifts associated with transient fluid swelling, elevated stromal extracellular matrix production, ${ }^{12,13}$ increased intraocular pressure with postoperative steroid use,${ }^{14}$ and corneal biomechanical changes are documented after surgical perturbation of the cornea. ${ }^{15,16}$ Increase in $\mathrm{Km}$ is a consequence of these suspected mechanisms of myopic shift.

After the first postoperative month, our results indicated a steady increase in $\mathrm{Km}$ from baseline alongside a hyperopic shift. Corneal topography showed annular steepening that occurred over the inlay body, and flattening over the inlay aperture, which includes the visual axis. It appears that this pattern caused the observed hyperopic shift. During the 36-month period, none of the eyes developed haze or scar formation to explain the corresponding hyperopic shift.

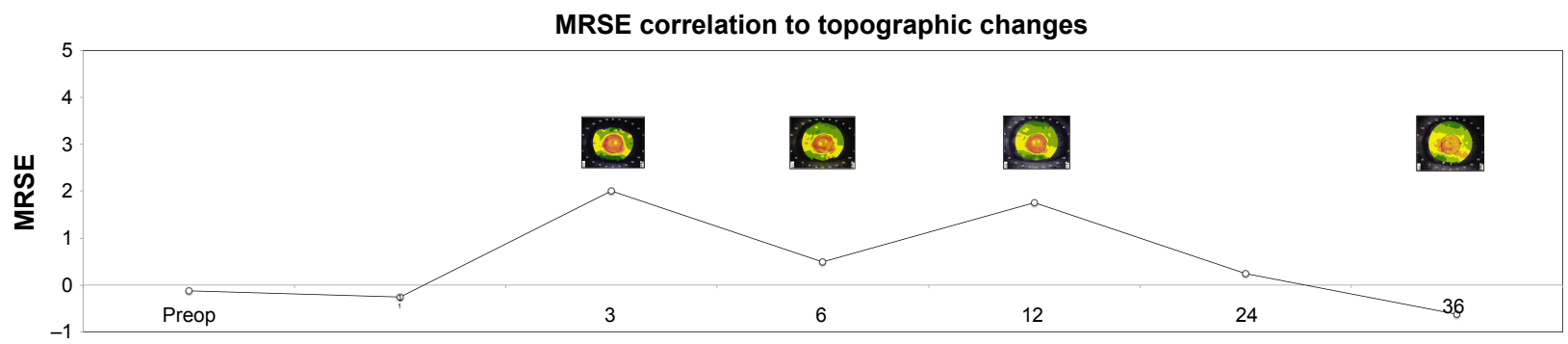

Postoperative time point (months)

Figure 6 Longitudinal corneal topographic changes associated with MRSE changes in a single patient. Pronounced correlation of topographic "ring" disappearance and myopic regression in late postoperative interval.

Note: The inset images represent the corneal topographical changes at the corresponding 3, 6, 12, and 36 postop months.

Abbreviations: MRSE, manifest refractive spherical equivalent; postop, postoperative; preop, preoperative. 


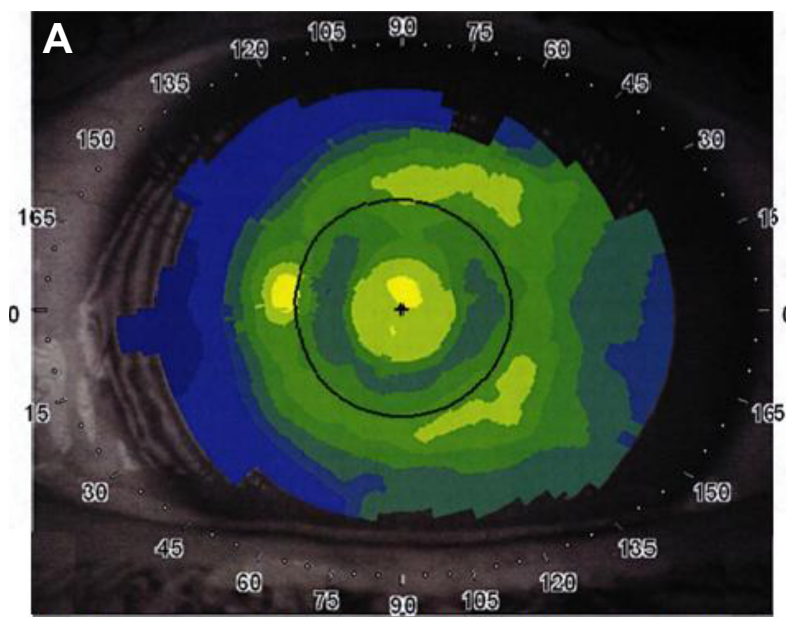

Central steepening and annular flattening with myopic shift

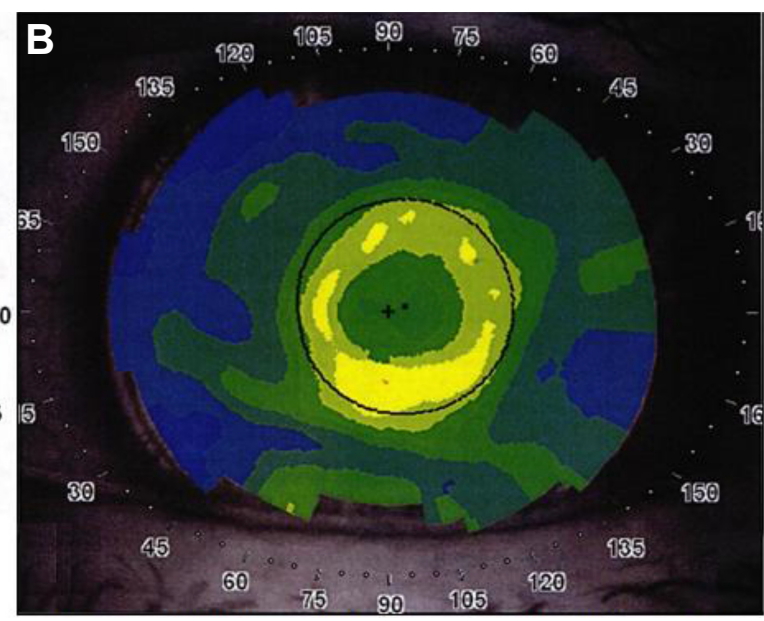

Central flattening and annular steepening with hyperopic shift

Figure 7 (A) Central steepening and annular flattening topography at 36 months in a patient with significant myopic shift. (B) Central flattening and annular steepening topography at 36 months in a patient with significant hyperopic shift.

Of note, two of the patients demonstrated an inverse pattern, with central steepening and peripheral flattening, with a corresponding myopic shift (Figure 4). Such pattern of epithelial and/or stromal remodeling was quite unique to these two patients, and we do not have a good explanation for it.

Similar ring topography patterns following KAMRA implantation have been described. ${ }^{8,9}$ Abbouda et al performed a prospective confocal microscopy study of 12 patients implanted with three models of the KAMRA inlay (ACI7000, seven cases; ACI7000T, one case; and ACI7000PDT, four cases). Concurrent keratocyte activation and decreased keratocyte density were noted around and above the inlay at 6 months. Four patients in the microscopy cohort required explant of their inlays (ACI7000, three patients; ACI7000T, one patient). A ring topography pattern similar to that observed in our study was seen in all explant patients. Annular steepening was not

Table 3 SIA between postoperative time points

\begin{tabular}{|c|c|c|c|c|}
\hline \multirow{3}{*}{$\begin{array}{l}\text { Postoperative } \\
\text { interval }\end{array}$} & \multicolumn{4}{|c|}{ Summated mean SIA vector (D) } \\
\hline & \multicolumn{2}{|c|}{$\begin{array}{l}\text { Keratometric } \\
\text { cylinder }\end{array}$} & \multicolumn{2}{|l|}{$\begin{array}{l}\text { Manifest } \\
\text { cylinder }\end{array}$} \\
\hline & Left eyes & Right eyes & Left eyes & Right eyes \\
\hline Preop-36 months & $0.35 \times 70^{\circ}$ & $0.28 \times 100^{\circ}$ & $0.42 \times 89^{\circ}$ & $0.35 \times 106^{\circ}$ \\
\hline Preop-I month & $0.22 \times 77^{\circ}$ & $0.26 \times 98^{\circ}$ & $0.22 \times 91^{\circ}$ & $0.08 \times 87^{\circ}$ \\
\hline I month-3 months & $0.11 \times 77^{\circ}$ & $0.11 \times 115^{\circ}$ & $0.06 \times 54^{\circ}$ & $0.10 \times 120^{\circ}$ \\
\hline 3 months -6 months & $0.04 \times 55^{\circ}$ & $0.15 \times 56^{\circ}$ & $0.08 \times 79^{\circ}$ & $0.14 \times 23^{\circ}$ \\
\hline 6 months -12 months & $0.10 \times 180^{\circ}$ & $0.12 \times 161^{\circ}$ & $0.03 \times 140^{\circ}$ & $0.03 \times 28^{\circ}$ \\
\hline 12 months -24 months & $0.11 \times 88^{\circ}$ & $0.08 \times 147^{\circ}$ & $0.17 \times 158^{\circ}$ & $0.10 \times 100^{\circ}$ \\
\hline 24 months -36 months & $0.07 \times 21^{\circ}$ & $0.08 \times 50^{\circ}$ & $0.16 \times 88^{\circ}$ & $0.27 \times 114^{\circ}$ \\
\hline
\end{tabular}

Abbreviations: preop, preoperative; SIA, surgically induced astigmatism. shown to be associated with hyperopic shift in that study, and the mean MRSE of explanted patients was $-0.1 \pm 1.0 \mathrm{D}$ (range 0 to -1.75 ). Although limited by sample size, no newer ACI7000PDT inlays were removed, suggesting an inverse association between lower inlay porosity and higher stromal keratocyte activity. ${ }^{8}$ Dexl et al noted a wound healing response in some patients characterized by steepening over the inlay and flattening over the aperture with a hyperopic shift similar to that seen in this study. However, the implanted inlay was a different model (ACI7000) and thicker than the one used in this study (ACI7000PDT). Moreover, it was implanted under a flap instead of an intrastromal pocket and was implanted at a shallower depth (170 vs $210 \mu \mathrm{m}){ }^{9}$

Beyond altering the satisfaction of inlay patients, the decoupling of keratometry and MRSE observed in our study may impact subsequent surgical interventions, such as cataract surgery. In many patients with notable ring steepening, the steepness over the visual axis was often misrepresented by the tomographer-reported $\mathrm{Km}$ by approximately $1 \mathrm{D}$ due to the inclusion of the annular steepening pattern over the inlay body. When elevated keratometry parameters are used as inputs to intraocular lens (IOL) prediction formulas, such as the Barrett Universal II Formula or the Hill RBF, the suggested IOL power may change significantly ( $\geq 1.0 \mathrm{D})$. Tan et al evaluated visual outcomes in two patients with a history of KAMRA inlay implantation who underwent biometry calculations and phacoemulsification surgery with the KAMRA inlay in place. ${ }^{17}$ Each patient had good IOL power predictability using the SRK/T formula with post-KAMRA biometry 
A

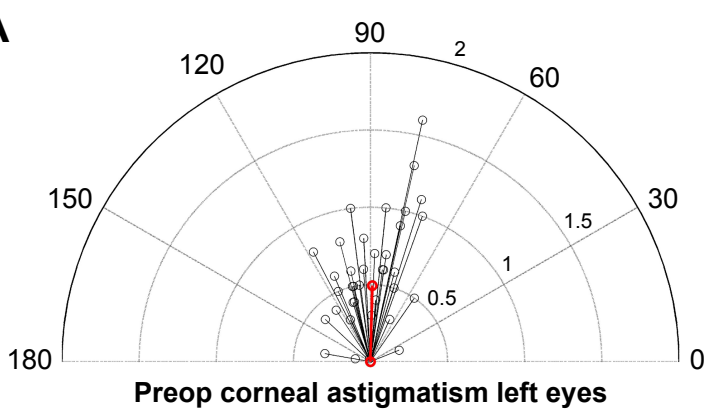

B

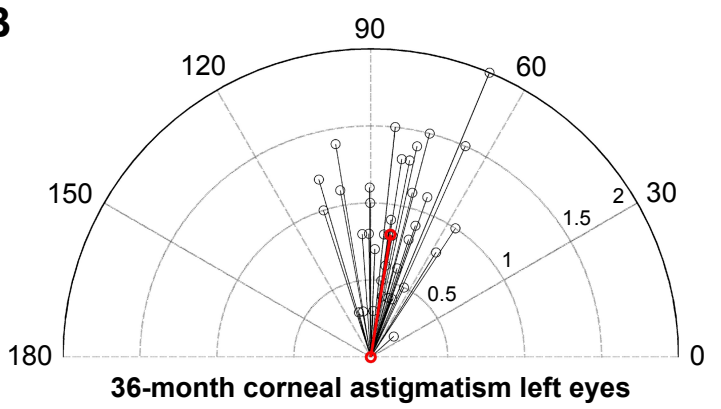

C

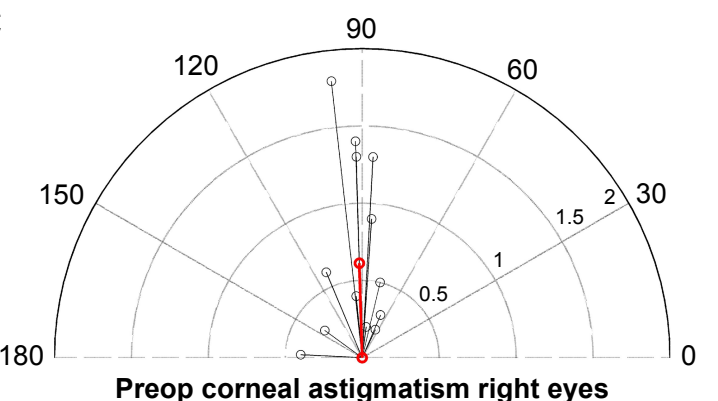

D

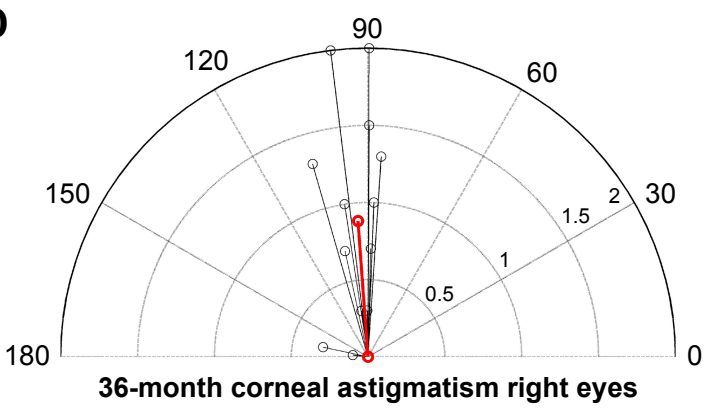

Figure 8 Depictions of corneal astigmatisms and axis for left and right eyes in vector format.

Notes: (A) Red line shows mean corneal astigmatism in corresponding axis preoperatively for left eyes; (B) red line shows mean corneal astigmatism in corresponding axis at 36 months postoperatively for left eyes; (C) red line shows mean corneal astigmatism in corresponding axis preoperatively for right eyes; (D) red line shows mean corneal astigmatism in corresponding axis at 36 months postoperatively for right eyes.

Abbreviation: preop, preoperative.

values as inputs, despite topographic evidence of mild ring steepening. The predicted power calculations differed from the actual power by $+0.34 \mathrm{D}$ and $-0.05 \mathrm{D} .{ }^{17}$ Additional cases of cataract surgeries after inlay implantation have been reported. ${ }^{18}$ In these cases, the Holladay 1 and SRK/T formulas were accurate in calculating the IOL power. The difference in target and achieved IOL power for these three cases was $+0.675 \mathrm{D},+0.10 \mathrm{D}$, and $+0.19 \mathrm{D} .{ }^{18}$ These cases show that the increased $\mathrm{Km}$ from the KAMRA inlay may affect the IOL calculations and target IOL power. Given that four of the cases were slightly hyperopic compared with the target refraction postoperatively, increasing the target IOL power by 0.25 or $0.50 \mathrm{D}$ is recommended or justifiable in patients with a history of KAMRA inlay.

Per FDA requirements to demonstrate biocompatibility, the KAMRA inlay has been shown to be noncytotoxic, nonsensitizing, nonirritating, nongenotoxic, and nonmutagenic in cellular assays. ${ }^{7}$ However, the smoldering keratocyte response as evidenced by persistent haze in many subjects in confocal studies ${ }^{8}$ suggests that the human cornea may never fully adapt to the long-term presence of the inlay. In one study, cellular apoptosis and CD11b+ levels in rabbits implanted with KAMRA inlays have been shown to be significantly elevated in the immediate postoperative period (24-48 hours) compared with control corneas subjected only to femtosecond laser pocket creation. By 6 weeks, apoptosis and CD11b+ levels were equivalent between the two groups. ${ }^{19}$ Long-term analyses of keratocyte distress in the peri-KAMRA stroma should be performed, given that topographical ring formation and associated clinically significant visual changes often did not emerge until beyond 12 months in this study.

Induction of corneal or refractive astigmatism as a result of inlay implantation appeared to be clinically unremarkable and congruent with the coupling effect of a temporal incision, in that steepening occurred in the vertical meridian. Vector analysis was performed in order to determine whether induced astigmatism was directionally biased due to the presence of the KAMRA inlay. The results demonstrated that there was no significant induction of astigmatism in any meridian, although stromal remodeling may occur in response to the presence of the inlay. Furthermore, if the KAMRA inlay were to induce a clinically significant amount of astigmatism, we would expect it to be correlated to the refractive astigmatism, and it was not.

\section{Conclusion}

From the long-term data in this study, we conclude that longitudinal keratometric and topographic changes may occur following small-aperture corneal inlay implantation, resulting 
in refractive shifts. These shifts may affect subsequent procedures, such as cataract surgery. Some patients may need subsequent treatments after KAMRA inlay, such as photo refractive keratectomy, for the correction of these refractive shifts.

\section{Acknowledgment}

Data gathering and analysis and preparation of the manuscript were funded by an unrestricted grant from Research to Prevent Blindness (RPB), 360 Lexington Avenue, Floor 22, New York, NY 10017, USA.

\section{Disclosure}

$\mathrm{PCH} \mathrm{Jr}$ and PCH own shares in AcuFocus Inc. PCH Jr is a consultant for AcuFocus. SHL has received travel expenses from AcuFocus Inc. in the past. The authors report no other conflicts of interest in this work.

\section{References}

1. Frick KD, Joy SM, Wilson DA, Naidoo KS, Holden BA. The Global Burden of Potential Productivity Loss from Uncorrected Presbyopia. Ophthalmology. 2015;122(8):1706-1710.

2. Arlt E, Krall E, Moussa S, Grabner G, Dexl A. Implantable inlay devices for presbyopia: the evidence to date. Clin Ophthalmol. 2015;9: 129-137.

3. Yılmaz OF, Alagöz N, Pekel G, et al. Intracorneal inlay to correct presbyopia: Long-term results. J Cataract Refract Surg. 2011;37(7): 1275-1281.

4. Seyeddain O, Hohensinn M, Riha W, et al. Small-aperture corneal inlay for the correction of presbyopia: 3-year follow-up. J Cataract Refract Surg. 2012;38(1):35-45.

5. Jalali S, Aus der Au W, Shaarawy T. AcuFocus Corneal Inlay to Correct Presbyopia Using Femto-LASIK. One Year Results of a Prospective Cohort Study. Klin Monbl Augenheilkd. 2016;233(4):360-364.
6. Dexl AK, Seyeddain O, Riha W, et al. One-year visual outcomes and patient satisfaction after surgical correction of presbyopia with an intracorneal inlay of a new design. J Cataract Refract Surg. 2012;38(2): 262-269.

7. United States Food and Drug. Administration Summary of Safety and Effectiveness Data (SSED) (P120023). Available from: https:// www.accessdata.fda.gov/cdrh_docs/pdf12/P120023B.pdf. Accessed August 8, 2017.

8. Abbouda A, Javaloy J, Alió JL. Confocal microscopy evaluation of the corneal response following AcuFocus KAMRA inlay implantation. J Refract Surg. 2014;30(3):172-178.

9. Dexl AK, Jell G, Strohmaier C, et al. Long-term outcomes after monocular corneal inlay implantation for the surgical compensation of presbyopia. J Cataract Refract Surg. 2015;41(3):566-575.

10. Naroo SA, Bilkhu PS, Shehzad AN, Paramdeep SB. Clinical utility of the KAMRA corneal inlay. Clin Ophthalmol. 2016;10:913-919.

11. Alpins N. Astigmatism analysis by the Alpins method. $J$ Cataract Refract Surg. 2001;27(1):31-49.

12. Erie JC. Corneal wound healing after photorefractive keratectomy: a 3-year confocal microscopy study. Trans Am Ophthalmol Soc. 2003; 101:293-333.

13. Rocha KM, Krueger RR. Spectral-domain optical coherence tomography epithelial and flap thickness mapping in femtosecond laser-assisted in situ keratomileusis. Am J Ophthalmol. 2014;158(2):293-301.

14. Bamashmus MA, Saleh MF. Post-LASIK interface fluid syndrome caused by steroid drops. Saudi J Ophthalmol. 2013;27(2):125-128.

15. Guirao A. Theoretical elastic response of the cornea to refractive surgery: risk factors for keratectasia. J Refract Surg. 2005;21(2):176-185.

16. Petroll WM, Roy P, Chuong CJ, et al. Measurement of Surgically Induced Corneal Deformations Using Three-Dimensional Confocal Microscopy. Cornea. 1996;15(2):154-164.

17. Tan T-E, Mehta JS. Cataract surgery following KAMRA presbyopic implant. Clin Ophthalmol. 2013;7:1899-1903.

18. Moshirfar M, Quist TS, Skanchy DF, et al. Cataract Surgery in Patients with a Previous History of KAMRA Inlay Implantation: A Case Series. Ophthalmol Ther. 2017;6(1):207-213.

19. Santhiago MR, Barbosa FL, Agrawal V, et al. Short-term Cell Death and Inflammation After Intracorneal Inlay Implantation in Rabbits. J Refract Surg. 2012;28(2):144-149.
Clinical Ophthalmology

\section{Publish your work in this journal}

Clinical Ophthalmology is an international, peer-reviewed journal covering all subspecialties within ophthalmology. Key topics include: Optometry; Visual science; Pharmacology and drug therapy in eye diseases; Basic Sciences; Primary and Secondary eye care; Patient Safety and Quality of Care Improvements. This journal is indexed on Submit your manuscript here: http://www.dovepress.com/clinical-ophthalmology-journal

\section{Dovepress}

PubMed Central and CAS, and is the official journal of The Society of Clinical Ophthalmology (SCO). The manuscript management system is completely online and includes a very quick and fair peer-review system, which is all easy to use. Visit http://www.dovepress.com/ testimonials.php to read real quotes from published authors. 